\title{
New perspective in the assessment of total intracellular magnesium
}

\author{
Azzurra Sargenti, ${ }^{1}$ Lucia Merolle, ${ }^{1}$ Giulia Andreani, ${ }^{2}$ Concettina Cappadone, ${ }^{1}$ \\ Giovanna Farruggia, ${ }^{1,3}$ Stefano lotti, ${ }^{2,3}$ Chiara Marraccini ${ }^{4}$ \\ ${ }^{1}$ Department of Pharmacy and Biotechnologies, University of Bologna; ${ }^{2}$ Department of Veterinary \\ Medical Sciences, University of Bologna; ${ }^{3}$ National Institute of Biostructures and Biosystems, \\ Roma; ${ }^{4}$ Department of Life Sciences, University of Modena and Reggio Emilia, Italy
}

\begin{abstract}
Magnesium (Mg) is essential for biological processes, but its cellular homeostasis has not been thoroughly elucidated, mainly because of the inadequacy of the available techniques to map intracellular Mg distribution. Recently, particular interest has been raised by a new family of fluorescent probes, diaza-18-crown-hydroxyquinoline (DCHQ), that shows remarkably high affinity and specificity for $\mathrm{Mg}$, thus permitting the detection of the total intracellular Mg. The data obtained by fluorimetric and cytofluorimetric assays performed with DCHQ5 are in good agreement with atomic absorption spectroscopy, confirming that DCHQ5 probe allows both qualitative and quantitative determination of total intracellular Mg.
\end{abstract}

\section{Introduction}

Magnesium (Mg) is essential for numerous biological processes, but its cellular homeostasis has not been thoroughly elucidated; yet, some peculiarities in its regulation are emerging, indicating that free and total Mg undergo two independent regulatory mechanism and therefore discriminating between the two pools is crucial. Atomic absorption spectroscopy (AAS) is the reference technique to measure total Mg content but requires volatilization of the sample and a high number of cells (several millions). Recently, particular interest has been raised by a new family of fluorescent probes, diaza-18-crownhydroxyquinoline (DCHQ), that shows remarkably high affinity and specificity for Mg. Specifically, DCHQ1 (Figure 1) is capable to image

Correspondence: Azzurra Sargenti, Department of Pharmacy and Biotechnologies, University of Bologna, via Belmeloro 6, 40126 Bologna, Italy. Tel./Fax: +39.051.2099700.

E-mail: azzurra.sargenti@unibo.it

Key words: total intracellular magnesium, biological process, DCHQ.

CC Copyright A. Sargenti et al., 2014

Licensee PAGEPress, Italy

Journal of Biological Research 2014; 87:2140

doi:10.4081/jbr.2014.2140

This article is distributed under the terms of the Creative Commons Attribution Noncommercial License (by-nc 3.0) which permits any noncommercial use, distribution, and reproduction in any medium, provided the original author(s) and source are credited. intracellular Mg in living cells and to assess total cellular in small sample, providing overlapping results with AAS. ${ }^{1}$ However, DCHQ1 showed some limitation, such us poor intracellular retention and a certain degree of fluorescence instability, invalidating its use, for instance, in time based measures of $\mathrm{Mg}$ cellular fluxes. In addition, DCHQ1 is excitable only in the UV range $(360 \mathrm{~nm})$. A new phenyl-derivate, DCHQ5 (Figure 1) is highly retained within cells and maintaining stable fluorescence intensity up to $30 \mathrm{~min}$ of light exposure. ${ }^{2}$ Furthermore, DCHQ5 displayed higher fluorescence enhancement upon Mg binding than DCHQ1, and is excitable also in the visible range. Aim of this study is to perform a quantitative assessment of total intracellular $\mathrm{Mg}$ by this new hydroxyiquinoline derivative chemosensor.

\section{Materials and Methods}

HL60 cells were maintained in Roswell Park Memorial Institute (RPMI) 1640 medium supplemented with $2 \mathrm{mM}$ L-Glutamine, 10\% foetal bovine serum (FBS) at $37^{\circ} \mathrm{C}$ and $5 \% \mathrm{C02}$. Cells were washed twice in PBS without $\mathrm{Ca}^{2+}$ and $\mathrm{Mg}^{2+}$, counted and resuspended at $5 \times 10^{5}$ cells/mL. For flow cytometric assays, cells resuspended at $5 \times 10^{5}$ cells/mL were incubated with DCHQ5 $5 \mu \mathrm{M}$ in phosphate buffered saline (PBS) without $\mathrm{Ca}^{2+}$ and $\mathrm{Mg}^{2+}$ for 15 min in the dark, counterstained with Propidium Iodide $5 \mu \mathrm{g} / \mathrm{mL}$ and analyzed by flow cytometry exciting DCHQ5 fluorescence at 360 or $488 \mathrm{~nm}$ and collecting on a logarithmic scale the fluorescence emission at $525 \mathrm{~nm}$. Samples were lysed by sonifier, and $200 \mu \mathrm{L}$ of the sample were added to $1.8 \mathrm{~mL}$ of DCHQ5 $15 \mu \mathrm{M}$ in methanol and oil production system (MOPS) 20 $\mathrm{mM} /$ Methanol 50\%. Fluorescence spectra were collected with ex 360 $\mathrm{nm} \mathrm{Mg}$ concentration was assessed comparing fluorescence intensity at em $510 \mathrm{~nm}$ of the samples with a calibration curve prepared with $\mathrm{MgSO}_{4}$. For AAS, the sonified samples were digested in HNO3 $1 \mathrm{~N}$, centrifuged and the supernatant analyzed by a spectrometer equipped with an air/acetylene flame.

\section{Results and Discussion}

In this work we tested the analytical capability of DCHQ5. The probe can be applied for the cytofluorimetric assay of intracellular total Mg, assessing intracellular variations of $\mathrm{Mg}$ content in different proliferative states. It is known that dimethyl sulfoxide (DMSO)-induced differentiation in HL60 promielocitic leukemic cells halves cellular Mg content, from an average of 25 to $12 \mathrm{nmol} / 10^{6}$ cell. ${ }^{1}$ DCHQ5 allowed to detect the Mg decrease in G0/G1 blocked cells, as reported in Figure 2. 
Flow cytometric assays, performed both by UV and visible excitation, showed a decrease of about $50 \%$ of the mean fluorescence intensity (evaluated as mean channel of the fluorescence distribution) of differentiated $v s$ control cells: in the experiment showed in Figure 2, for example, the geometric mean channel of control cells and DMSO differentiated cells were respectively 94 and 45 with Visible excitation and 83 and 43 with UV excitation.

Therefore, we planned a protocol to quantitatively assess total intracellular Mg in sonicated cellular samples by using a simple spectrofluorimetric assay. We compared the data obtained by DCHQ5 with AAS, the reference technique for the quantification of total intracellular Mg (Table 1).

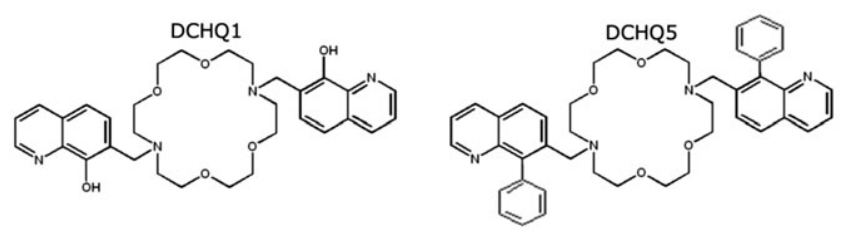

Figure 1. Structure of the DCHQ1 e DCHQ5 probes.
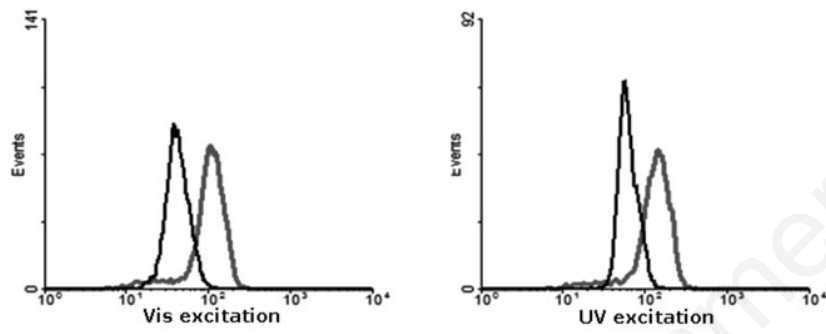

Figure 2. DCHQ5 fluorescence distribution of control (grey line), and dimethyl sulfoxide-differentiated (black line) HL60 cells.
Table 1. Quantification of total intracellular magnesium in different samples by DCHQ 5 and atomic absorption spectroscopy.

\begin{tabular}{lcc}
$\begin{array}{l}\text { Sample at different } \\
\text { passage }\end{array}$ & $\begin{array}{c}\text { DCH05 } \\
\text { Mg }\left(\mathrm{nmol} / 10^{6} \text { cells }\right) \mathrm{Mg}\left(\mathrm{Amol} / 10^{6} \text { cells }\right)\end{array}$ \\
HL60 121p & $19.75 \pm 0.76$ & $22.78 \pm 0.38$ \\
HL60 124p & $22.93 \pm 1.30$ & $20.70 \pm 0.61$ \\
\hline HL60 128p & $23.77 \pm 1.14$ & $24.70 \pm 0.89$ \\
HT29 67p & $24.31 \pm 1.13$ & $23.94 \pm 0.89$ \\
\hline HT29 69p & $23.96 \pm 0.37$ & $21.17 \pm 0.71$ \\
\hline
\end{tabular}

AAS, atomic absorption spectroscopy.

The data obtained by DCHQ5 are in good agreement with atomic absorption spectroscopy, confirming that DCHQ5 probe allows both qualitative and quantitative determination of total intracellular Mg.

\section{Conclusions}

In conclusion, we demonstrate that DCHQ5 can be considered the future reference molecular probe for the study of intracellular Mg homeostasis, broadening the field of application of DCHQ chemosensors.

\section{References}

1. Farruggia G, Iotti S, Prodi L, et al. 8-hydroxyquinoline derivatives as fluorescent sensors for magnesium in living cells. J Am Chem Soc 2006;128:344-50.

2. Farruggia G, Iotti S, Prodi L, et al. Hydroxyquinoline derivatives as fluorescent probes to measure intracellular magnesium: insight into characteristics and biological applications. J Fluoresc 2009;1911-9. 